\title{
Síntomas del tracto urinario inferior en la mujer y afectación de la calidad de vida. Resultados de la aplicación del King’s Health Questionnaire
}

\author{
Espuña Pons M*, Puig Clota M.
}

*Institut Clinic de Ginecología, Obstetricia i Neonatología. Hospital Clínic. Universidad de Barcelona.

Actas Urol Esp. 2006;30(7):684-691

\section{RESUMEN}

SÍNTOMAS DEL TRACTO URINARIO INFERIOR EN LA MUJER Y AFECTACIÓN DE LA CALIDAD DE VIDA. RESULTADOS DE LA APLICACIÓN DEL KING'S HEALTH QUESTIONNAIRE

Fundamento: La auto-evaluación de la calidad de vida (CV) de las mujeres con síntomas urinarios puede ayudar a decidir la estrategia de tratamiento más adecuada en cada caso.

Método: Estudio epidemiológico, observacional, transversal y multicéntrico de 674 mujeres que acudieron a una consulta de ginecología con sintomas sugestivos de Vejiga Hiperactiva, con o sin incontinencia urinaria(IU). Todas ellas cumplimentaron por escrito el King’s Health Questionnaire(KHQ). Además se recogieron datos sociodemográficos y un registro de los síntomas urinarios de la dimensión de síntomas del KHQ y el grado de afectación que producían.

Resultados: Los síntomas más frecuentes fueron "aumento de frecuencia miccional” (612 mujeres-90,8\%), seguido de "urgencia” (562-83,4\%), "nocturia” (543-80,6\%) e "IU de Esfuerzo" (535-79,4\%). Los sintomas "frecuencia”, "nocturia”, "urgencia” e "IU por urgencia" fueron más frecuentes en las mujeres de 65 o más años y el de "IU de esfuerzo" en las menores de esa edad (79,8\% vs 77,9\%). 210 mujeres no cumplimentaron todas las dimensiones del KHQ, siendo las menos contestadas: “Limitaciones Físicas"(35,7\%), “Relaciones Personales”(29,5\%), “Limitaciones Sociales"(29\%) e “Impacto de la IU”(28,6\%). La puntuación total del KHQ fue 38,3 (DE=19,2). Las mayores puntuaciones (peor CV) corresponden a "Impacto de la IU", "Afectación por Problemas Urinarios", "Limitaciones Físicas" y "Limitaciones en las Actividades Cotidianas”. Las variables asociadas a la puntuación global del KHQ fueron (regresión lineal múltiple): edad, IMC, IU por urgencia, IU en el acto sexual, infecciones frecuentes en vías urinarias y otros problemas urinarios.

Conclusiones: La afectación de la CV en las mujeres con síntomas urinarios es importante. Los síntomas que más contribuyen a la afectación de la CV son la "IU en el acto sexual", la "IU de urgencia" y las "infecciones frecuentes en vías urinarias".

Palabras clave: Sintomas urinarios. Vejiga Hiperactiva. Calidad de Vida. Mujer.

\section{ABSTRACT \\ LOWER URINARY TRACT SYMPTOMS IN WOMEN AND IMPACT ON QUIALITY OF LIFE. RESULTS OF THE APPLICATION OF THE KING'S HEALTH QUESTIONNAIRE}

Background: The self-assessment of quality of life (QoL) of women with urinary symptoms may help in selecting the best treatment in each case.

Method: Epidemiologic, observational, cross-sectional and multicentric study of 674 women who underwent to a gynecology unit with symptoms suggesting Overactive Bladder, with or without urinary incontinence(UI). All women fill out the King's Health Questionnaire. Sociodemographic data and a complete register of urinary symptoms and the degree of afectation which caused, were also collected.

Results: Most frequent symptoms were "frequency" (612 women-90.8\%), followed by "urgency" (562-83.4\%), "nocturia" (543-80.6\%) and "stres UI" (535-79.4\%). Symptoms of "frecuency", "nocturia”, "urgency" and "urgency UI" were more frequent in women aged 65 or under 65 years and that of "stress UI", in women over 65 years (79.8\% vs $77.9 \%) .210$ women did not fill out all the KHQ dimensions, mainly "Personal Limitations", "Personal Relationship", "Social limitations" and "Incontinence Impact". Global KHQ score was38.3 (SD=19.2). Higher scores (worse QoL) corresponded to "Incontinence Impact”, "Severity Measures", "Personal Limitations" and "Role Limitations". Variables associated to global KHQ score were (multiple linear regression): age, BMI, urgency UI, UI in sexual intercourse, frequent urinary infections.

Conclusions: QoL impact in women with urinary symptoms is important. The symptoms with higher association with GoL are: UI in sexual intercourse, urgency UI and frequent urinary infections.

Keywords: Urinary symptoms. Overactive Bladder. Quality of Life. Female. 
$\mathrm{L}$ os sintomas de incontinencia urinaria (IU) tienen una alta prevalencia en la población general de mujeres en España. Se estima que entre el 20\% y el 50\% de las mujeres pueden tener algún escape accidental de orina, aunque estas cifras, siendo reales, no son un reflejo de la cantidad de mujeres que realmente necesitan ayuda médica por su problema de IU. Existe un interés creciente entre los profesionales de la salud de realizar un diagnóstico y tratamiento adecuados y completos a las pacientes con IU, no obstante para que los recursos existentes se dirijan a las personas que realmente necesitan ayuda, es fundamental identificar aquellas que ven su vida afectada de forma importante por este problema.

Es fundamental antes de iniciar un tratamiento, conocer el tipo de síntomas que refiere la paciente, ya que los tratamientos son distintos según se trate de una paciente con una incontinencia urinaria de esfuerzo (IUE), una incontinencia urinaria de urgencia (IUU) o una incontinencia urinaria mixta (IU Mixta). Hay que tener en cuenta además que algunas pacientes refieren una asociación de síntomas urinarios con significado clínico y lo que la International Continence Society (ICS), en su nueva estandarización de terminología, denomina síndromes. Entre ellos el que se denomina "síndrome de Vejiga Hiperactiva" (VH) y que se define como "urgencia", a menudo asociada a aumento de frecuencia y/o nocturia, con o sin incontinencia de urgencia. El término de $\mathrm{VH}$ sólo se utiliza en aquellas pacientes en las que se ha demostrado que no existe infección de orina u otra patología orgánica que pueda ser la causa de sus síntomas urinarios.

La Internacional Consultation on Incontinence (ICI) señala que, en las pacientes con sintomas urinarios, la evaluación de la presencia o ausencia del síntoma por sí sola, no evalúa el impacto que este problema produce en su vida y que la forma más adecuada de evaluar los síntomas y la afectación de la calidad de vida, es recogiendo la opinión del paciente a través de la auto-cumplimentación de cuestionarios de evaluación robustos desde el punto de vista de la psicometría ${ }^{1}$. Así pues el impacto que los síntomas urinarios producen en la calidad de vida de las mujeres, dependen de: el tipo de sintomas, de la gravedad de los mismos y del grado de afectación que estos producen en ella de acuerdo a sus características individuales (edad, tipo de actividad en la que se siente limitada, etc.). Los sintomas de IUU han demostrado tener en algunos estudios un impacto mayor en la calidad de vida que los demás tipos de $\mathrm{IU}^{2}$.

En la práctica asistencial, dado que la IU es una disfunción que no supone una amenaza vital para la persona que la padece, es fundamental adaptar el tratamiento a las necesidades de la paciente. La aplicación de cuestionarios autocumplimentados específicos de calidad de vida puede permitir valorar las mujeres con síntomas urinarios, evaluando simultáneamente el tipo de sintomas y el grado de afectación de su calidad de vida y según la información obtenida decidir la estrategia de tratamiento más adecuada no sólo por el tipo de síntomas sino por la afectación que éstos producen en la vida de esta paciente ${ }^{3}$. Entre los cuestionarios existentes que evalúan sintomas y calidad de vida, el King's Health Questionnaire (KHQ) es probablemente el más utilizado y se dispone de una versión validada adecuadamente en España ${ }^{4}$. El estudio de validación de la versión española del cuestionario fue realizado en una muestra limitada de mujeres $(\mathrm{N}=162)$, con diferentes tipos de IU de acuerdo a la prueba urodinámica y que acudieron a una de las 23 consultas de urología de España participantes en el estudio.

En el presente estudio se utilizó la versión española del King's Health Questionnaire en un grupo poblacional grande de mujeres que acudieron a una consulta ginecológica con sindrome de $\mathrm{VH}$ (sintomas de urgencia-frecuencia, asociados o no a otros síntomas urinarios), con el objetivo de evaluar la afectación de su calidad de vida en un grupo amplio de mujeres en nuestro ámbito ginecológico y analizar que síntomas están más relacionados con un mayor impacto en la @oL.

\section{PACIENTES Y MÉTODOS}

Estudio epidemiológico, observacional, transversal y multicéntrico en el que se incluyeron mujeres mayores de 18 años y sin límite superior de edad, con síntomas sugestivos de $\mathrm{VH}$, o lo que es lo mismo, síndrome de urgencia-frecuencia 
(que refieran frecuencia miccional superior a las 8 micciones en 24 horas y episodios de urgencia miccional), con o sin incontinencia. El único motivo de exclusión era no estar capacitada psíquicamente para responder a las preguntas formuladas durante la encuesta, hecho que ocurrió en dos pacientes. Se reclutaron un total de 891 pacientes en 228 consultas especializadas de ginecología. De éstas se excluyeron para el análisis 217 pacientes por los siguientes motivos (se podía indicar más de un motivo): 7 pacientes por defectos en el cuaderno de recogida de datos y 210 pacientes por no completar todo el cuestionario KHQ. Se presentan resultados de las restantes 674 mujeres que acudieron a una consulta ginecológica con sintomas urinarios. A todas las pacientes se les entregó un cuestionario de calidad de vida específico para la incontinencia de orina, el King's Health Questionnaire (KHQ) para ser auto-cumplimentado por escrito. Todas las mujeres eran mayores de 18 años de edad y prestaron consentimiento por escrito para su participación en el estudio.

El King's Health Questionnaire (KHQ) es un instrumento de evaluación específico de la calidad de vida en mujeres con $\mathrm{IU}^{5}$ que consta de 21 items distribuidos en 9 dimensiones: Percepción del Estado de Salud General-ESG (1 item), Afectación por Problemas Urinarios-APU (1 ítem), Limitaciones en las Actividades Cotidianas-LAC (2 items), Limitaciones Sociales-LS (2 items), Limitaciones Físicas-LF (2 items), Relaciones Personales-RP (3 items), Emociones-E (3 items), Sueño/Energía-SE (2ítems) e Impacto de la IUImIU (5 items). Cada ítem tiene una escala de respuesta tipo Likert con 4 posibles respuestas. El rango de puntuaciones de cada dimensión va de 0 (menor impacto de la IU y por tanto mejor calidad de vida) a 100 (mayor impacto, peor calidad de vida). La dimensión Relaciones Personales puede no cumplimentarse si la paciente considera que no proceden las preguntas, dado que se refieren a la vida de pareja. La versión española de este cuestionario ha demostrado tener adecuadas validez y fiabilidad para evaluar la calidad de vida de las mujeres con diferentes tipos de $\mathrm{IU}^{4}$.

Junto con el KHQ se recogieron datos sociodemográficos (edad, peso e IMC), un completo registro de los sintomas urinarios mediante la dimensión de síntomas del King’s Health Questionnaire (frecuencia, nocturia, urgencia, IU de urgencia, IU de esfuerzo, incontinencia con el acto sexual, infecciones en vías urinarias, dolor en la vejiga, dificultad al orinar y otros problemas urinarios) así como el grado de afectación (un poco, moderadamente, mucho).

Se analizaron la frecuencia de presentación de los diferentes síntomas urinarios así como la distribución de las pacientes de acuerdo al nivel de afectación de cada sintoma y a la edad. Respecto a las puntuaciones en las diferentes dimensiones del cuestionario KHQ, se analizaron la distribución de las mismas en la muestra global y separadas de acuerdo a los síntomas expresados por las mujeres. Por último se elaboró un modelo de regresión lineal múltiple para la puntuación global del cuestionario con las variables de síntomas expresados por las pacientes, la edad y el IMC.

\section{RESULTADOS}

Se estudiaron un total de 891 pacientes, a todas las cuales se les había hecho entrega del cuestionario en la consulta ginecológica. De éstas se consideraron no valorables a 217 mujeres. Así se presentan resultados de 674 mujeres valorables que representan el 75,5\% del total. La edad media (Desviación Estándar) de las pacientes fue de $56,1(10,8)$ años, el peso medio de $68,7(10,8)$ y el IMC medio de $26,7(4,3)$.

La distribución de los diferentes sintomas urinarios en la muestra estudiada se presenta en la Tabla 1, junto con el grado de afectación (un poco, moderadamente o mucho) que producía cada uno de ellos en las mujeres que lo padecían. El síntoma más referido fue el aumento de frecuencia miccional, seguido de urgencia, nocturia e IU de esfuerzo.

La distribución de los principales síntomas urinarios respecto de la edad en grupos se presentan en la Tabla 2 junto con la frecuencia, dentro de cada subgrupo, de las mujeres a las que los sintomas molestaban mucho. Agrupando a las mujeres en 2 grupos según tengan menos de 65 ó 65 o más años, se puede observar que los sintomas Frecuencia, Nocturia, Urgencia e IU por Urgencia son más frecuentes en el grupo de más edad, mientras que el síntoma de IU por Esfuerzo es más frecuente en el grupo de menor edad $(79,8 \%$ vs $77,9 \%)$. 
Tabla 1

Prevalencia de los distintos síntomas urinarios recogidos en el King’s Health Questionnaire y distribución de las pacientes que los refieren según el grado de afectación que producen

\begin{tabular}{|c|c|c|c|c|c|c|c|c|}
\hline & \multicolumn{2}{|c|}{ Incidencia } & \multicolumn{2}{|c|}{$\begin{array}{l}\text { Afectan } \\
\text { "un poco" }\end{array}$} & \multicolumn{2}{|c|}{$\begin{array}{c}\text { Afectan } \\
\text { "moderadamente" }\end{array}$} & \multicolumn{2}{|c|}{$\begin{array}{l}\text { Afectan } \\
\text { "mucho" }\end{array}$} \\
\hline & $\mathbf{n}$ & $\%^{(1)}$ & $\mathbf{n}$ & $\%^{(2)}$ & $\mathbf{n}$ & $\%^{(2)}$ & $\mathbf{n}$ & $\%^{(2)}$ \\
\hline Frecuencia & 612 & $90,8 \%$ & 105 & $17,2 \%$ & 297 & $48,5 \%$ & 210 & $34,3 \%$ \\
\hline Nocturia & 543 & $80,6 \%$ & 190 & $35,0 \%$ & 251 & $46,2 \%$ & 102 & $18,8 \%$ \\
\hline Urgencia & 562 & $83,4 \%$ & 139 & $24,7 \%$ & 218 & $38,8 \%$ & 205 & $36,5 \%$ \\
\hline I U de urgencia & 519 & $77,0 \%$ & 159 & $30,6 \%$ & 204 & $39,3 \%$ & 156 & $30,1 \%$ \\
\hline I U de esfuerzo & 535 & $79,4 \%$ & 163 & $30,5 \%$ & 216 & $40,4 \%$ & 156 & $29,2 \%$ \\
\hline I U en el acto sexual & 212 & $31,5 \%$ & 136 & $64,2 \%$ & 64 & $30,2 \%$ & 12 & $5,7 \%$ \\
\hline Infecciones en vías urinarias & 333 & $49,4 \%$ & 184 & $55,3 \%$ & 115 & $34,5 \%$ & 34 & $10,2 \%$ \\
\hline Dolor en la vejiga & 270 & $40,1 \%$ & 155 & $57,4 \%$ & 90 & $33,3 \%$ & 25 & $9,3 \%$ \\
\hline Dificultad al orinar & 194 & $28,8 \%$ & 120 & $61,9 \%$ & 56 & $28,9 \%$ & 18 & $9,3 \%$ \\
\hline Otros problemas urinarios & 35 & $5,2 \%$ & 13 & $37,1 \%$ & 16 & $45,7 \%$ & 6 & $17,1 \%$ \\
\hline
\end{tabular}

(1): Porcentaje de afectados con el problema urinario sobre el total de valorables $(n=674)$.

(2): Porcentaje de casos con el grado de afectación sobre el total de casos con el problema.

Tabla 2

Prevalencia de los síntomas urinarios recogidos en el King's Health Questionnaire en los diferentes grupos de edad y frecuencia de aparición del síntoma "con mucha molestia", en cada grupo

\begin{tabular}{|c|c|c|c|c|c|c|}
\hline \multicolumn{2}{|c|}{ Grupo de edad } & \multirow{2}{*}{$\begin{array}{c}\text { Frecuencia } \\
17(85 \%)\end{array}$} & \multirow{2}{*}{$\begin{array}{l}\text { Nocturia } \\
15(75 \%)\end{array}$} & \multirow{2}{*}{$\begin{array}{l}\text { Urgencia } \\
15(75 \%)\end{array}$} & \multirow{2}{*}{$\begin{array}{c}\text { IUU } \\
15(75 \%)\end{array}$} & \multirow{2}{*}{$\frac{\text { IUE }}{11(55 \%)}$} \\
\hline$<35$ años & n (\%) & & & & & \\
\hline$(\mathrm{N}=20)$ & M. $\mathrm{M}^{(1)}$ & $29,4 \%$ & $20 \%$ & $60 \%$ & $20 \%$ & $18,2 \%$ \\
\hline 35-45 años & n (\%) & $63(92,6 \%)$ & 53 (77,9\%) & $58(85,3 \%)$ & 51 (75\%) & 51 (75\%) \\
\hline$(\mathrm{N}=68)$ & M.M $\mathrm{M}^{(1)}$ & $33,3 \%$ & $20,8 \%$ & $32,8 \%$ & $25,5 \%$ & $31,4 \%$ \\
\hline 45-55 años & n (\%) & $187(88,2 \%)$ & $157(74,1 \%)$ & $168(79,2 \%)$ & $152(71,7 \%)$ & 177 (83,5\%) \\
\hline$(\mathrm{N}=212)$ & M.M. $\mathrm{M}^{(1)}$ & $6,7 \%$ & $15,3 \%$ & $32,7 \%$ & $25 \%$ & $26,6 \%$ \\
\hline 55-65 años & n (\%) & $200(91,3 \%)$ & 184 (84\%) & $181(82,6 \%)$ & 173 (79\%) & $175(79,9 \%)$ \\
\hline$(\mathrm{N}=219)$ & M.M $\mathrm{M}^{(1)}$ & $36 \%$ & $16,8 \%$ & $37 \%$ & $29,5 \%$ & $25,7 \%$ \\
\hline 65-75 años & n (\%) & $121(94,5 \%)$ & $109(85,2 \%)$ & $114(89,1 \%)$ & $103(80,5 \%)$ & $103(80,5 \%)$ \\
\hline$(\mathrm{N}=128)$ & M.M. $\mathrm{M}^{(1)}$ & $41,3 \%$ & $24,8 \%$ & $38,6 \%$ & $39,8 \%$ & $38,8 \%$ \\
\hline$\geq 75$ años & n (\%) & $24(92,3 \%)$ & 24 (92,3\%) & 26 (100\%) & $25(96,2 \%)$ & $17(65,4 \%)$ \\
\hline$(\mathrm{N}=26)$ & M.M $\mathrm{M}^{(1)}$ & $50 \%$ & $25 \%$ & $42,3 \%$ & $40 \%$ & $35,3 \%$ \\
\hline $\mathrm{p}^{(2)}$ & & 0,3685 & 0,0336 & 0,0168 & 0,0472 & 0,0250 \\
\hline
\end{tabular}

(1): Mucha Molestia (2): Test Chi-cuadrado

Del total de 891 mujeres, $210(23,6 \%)$ no cumplimentaron todas las dimensiones del cuestionario, siendo lo más frecuente el no responder a 1 dimensión $(58,6 \%)$ o a $2(20,5 \%)$. Casi un tercio de estas 210 mujeres dejaron sin cumplimentar alguna o varias de las siguientes dimensiones: Limitaciones Físicas (35,7\%), Relaciones Persona- les $(29,5 \%)$ aún considerando "no procede" como respuesta, Limitaciones Sociales (29\%) e Impacto de la IU $(28,6 \%)$. Respecto del total de la muestra estudiada ( $\mathrm{N}=891)$ la dimensión que presentó un tanto por ciento superior de mujeres que no respondieron a alguno de sus ítems fue Limitaciones Físicas $(8,4 \%)$, seguida de Relaciones Personales 
(6,9\%), Limitaciones Sociales $(6,8 \%)$ e Impacto de la IU $(6,7 \%)$. Además en la dimensión Relaciones Personales no se obtuvo puntuación, al no proceder su respuesta por no mantener relaciones sexuales, en 182 casos, por lo que los datos de esta dimensión y de la puntuación global se limitan a 492 casos. La puntuación media global del cuestionario $\mathrm{KHQ}$ fue de 38,3 (DE=19,2). Las puntuaciones de las dimensiones y la global del cuestionario se presentan en la Figura 1. En ella puede verse que los puntuaciones más altas (peor calidad de vida) corresponden a las dimensiones Impacto de la IU (ImIU), Afectación por Problemas Urinarios (APU), Limitaciones Físicas (LF) y Limitaciones en las Actividades Cotidianas (LAC).

En la Tabla 3 se presentan las puntuaciones medias y desviaciones estándar de las dimensiones y puntuación global del KHQ separadas en grupos de acuerdo a la presencia de cada uno de los síntomas estudiados. Dentro de cada grupo la puntuación global del KHQ fue mayor (peor calidad de vida) en los pacientes con el sintoma que en los que no lo presentaban $(\mathrm{p}<0,05$; análisis no presentado).

$\mathrm{El}$ análisis de regresión lineal múltiple realizado para la puntuación global del cuestionario KHQ se presenta en la Tabla 4. En ella puede

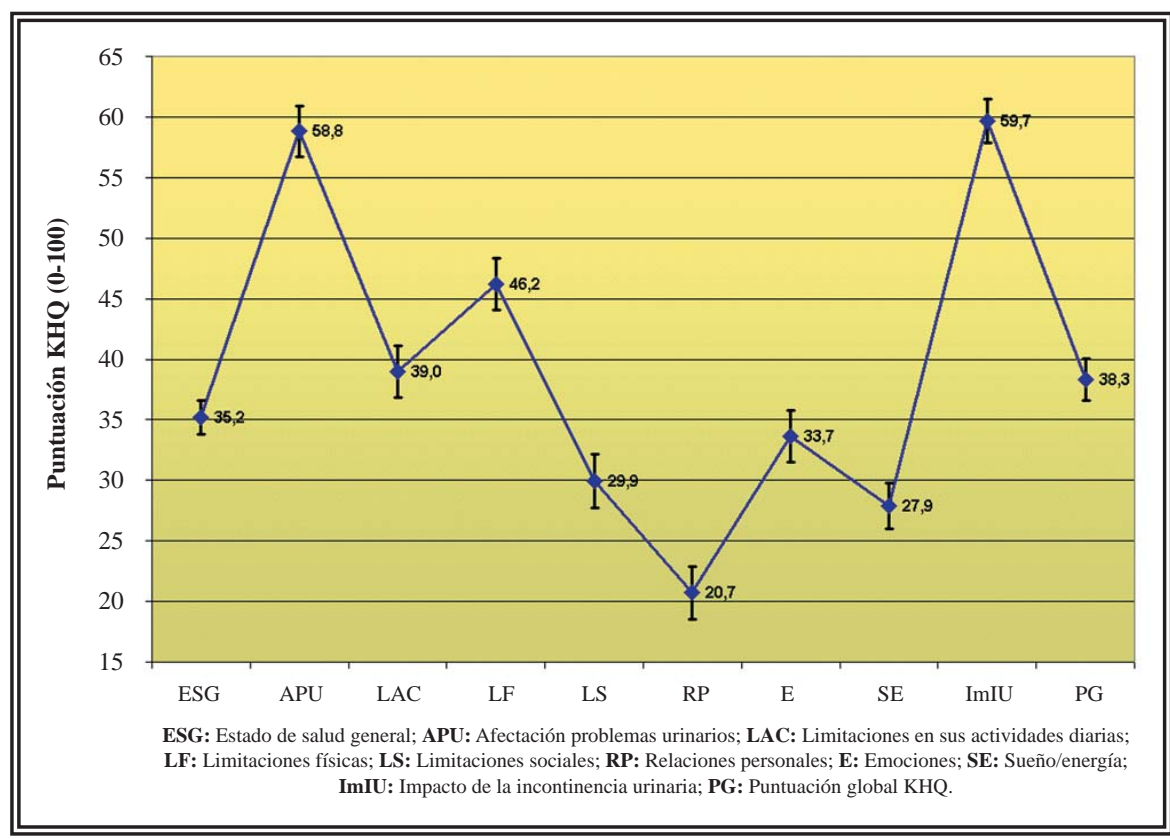

FIGURA 1: Puntuación del cuestionario KHQ para el global de la muestra. verse que el síntoma con mayor asociación independiente con la calidad de vida medida por el $\mathrm{KHQ}$ es la incontinencia en el acto sexual. La ecuación que resume el modelo calculado es la siguiente: Puntuación global KHQ $=85,8-1,4$ (edad) $-2,8($ IMC) $+0,1($ edad*IMC) $+6,4$ (incontinencia por urgencia) $+11,0$ (incontinencia en el acto sexual) $+5,4$ (infecciones frecuentes en vías urinarias) $+7,8$ (otros problemas urinarios).

\section{DISCUSIÓN}

El cuestionario KHQ es un cuestionario útil en la identificación de síntomas por parte de las pacientes que padecen IU y en cuantificar el impacto que producen en su calidad de vida. Las mujeres con sintomas de VH refieren en la mayoría de casos otros sintomas asociados. Los diferentes sintomas urinarios analizados por separado no parecen expresar diferentes perfiles de afectación de la calidad de vida, evaluada por el cuestionario KHQ. La IU en el acto sexual y la IU por urgencia se asocian a peor calidad de vida evaluada por el cuestionario KHQ.

A pesar de la utilidad del $\mathrm{KHQ}$ en estudios como el presente, el hecho de que una cuarta parte de las pacientes no cumplimentase todos los ítems, junto con la longitud de su extensión, aconsejan la utilización de otros cuestionarios más sencillos en la práctica clínica habitual, reservando el uso del KHQ para la investigación.

Los síntomas más frecuentemente señalados por las pacientes a través de la autocumplimentación del KHQ, de acuerdo a lo presentado en la Tabla 1, fueron en este orden: "frecuencia", "urgencia", "nocturia", "IU de esfuerzo" e "IU de urgencia". El número de mujeres con el nivel máximo de afectación por el síntoma fue superior en el caso de "urgencia" seguido por los sintomas "frecuencia", "IU de urgencia", "IU de esfuerzo" y "nocturia" en este orden. La mayor prevalencia de los sín- 
Tabla 3

Puntuaciones del cuestionario King's Health Questionnaire de acuerdo a la presencia de los distintos síntomas urinarios

\begin{tabular}{|c|c|c|c|c|c|c|c|c|c|c|c|c|c|c|c|}
\hline & \multicolumn{3}{|c|}{ Frecuencia } & \multicolumn{3}{|c|}{ Nocturia } & \multicolumn{3}{|c|}{ Urgencia } & \multicolumn{3}{|c|}{ IUU } & \multicolumn{3}{|c|}{ IUE } \\
\hline & $\mathrm{N}^{(\mathbf{1})}$ & Media & D.E. & $\mathbf{N}^{(1)}$ & Media & D.E. & $\mathbf{N}^{(\mathbf{1})}$ & Media & D.E. & $\mathbf{N}^{(\mathbf{1})}$ & Media & D.E. & $\mathbf{N}^{(\mathbf{1})}$ & Media & D.E. \\
\hline ESG & 612 & 35,2 & 18,9 & 543 & 36,3 & 19,1 & 562 & 35,7 & 19,2 & 519 & 35,7 & 19,2 & 535 & 35,7 & 18,9 \\
\hline APU & 612 & 59,6 & 27,5 & 543 & 61,5 & 27,1 & 562 & 60,6 & 27,6 & 519 & 60,8 & 27,8 & 535 & 59,4 & 28,0 \\
\hline LAC & 612 & 39,6 & 28,6 & 543 & 41,4 & 28,6 & 562 & 40,9 & 28,4 & 519 & 41,6 & 28,3 & 535 & 39,7 & 28,5 \\
\hline LF & 612 & 47,1 & 28,6 & 543 & 47,4 & 28,9 & 562 & 47,3 & 28,5 & 519 & 47,8 & 28,3 & 535 & 49,3 & 28,2 \\
\hline LS & 612 & 30,8 & 29,5 & 543 & 31 & 29,3 & 562 & 31,9 & 29,6 & 519 & 33,2 & 29,9 & 535 & 30,8 & 30,0 \\
\hline $\mathbf{R P}$ & 447 & 21,3 & 24,6 & 399 & 21,4 & 24,7 & 402 & 21,7 & 24,9 & 371 & 22,2 & 25,1 & 401 & 21,8 & 25,1 \\
\hline $\mathbf{E}$ & 612 & 34,4 & 28,4 & 543 & 35,9 & 28,5 & 562 & 35,2 & 28,7 & 519 & 36,1 & 28,9 & 535 & 34,5 & 28,7 \\
\hline SE & 612 & 29,1 & 25,5 & 543 & 31,8 & 25,0 & 562 & 30,3 & 25,6 & 519 & 31,0 & 26,0 & 535 & 28,3 & 25,7 \\
\hline mIU & 612 & 60,5 & 23,4 & 543 & 61,1 & 23,6 & 562 & 61,1 & 23,0 & 519 & 61,7 & 23,2 & 535 & 61,2 & 23,2 \\
\hline PG & 447 & 38,9 & 19,3 & 399 & 40,2 & 19,3 & 402 & 40,0 & 19,4 & 371 & 40,7 & 19,1 & 401 & 39,3 & 19,5 \\
\hline
\end{tabular}

ESG: Percepción del Estado de Salud General, APU: Afectación por Problemas Urinarios, LAC: Limitaciones en las Actividades Cotidianas, LS: Limitaciones Sociales, LF: Limitaciones Físicas, RP: Relaciones Personales, E: Emociones, SE: Sueño/Energía, ImIU: Impacto de la IU, PG: Puntuación Global.

(1) No todas las pacientes aportaron el dato de la dimensión RP.

Tabla 4

Análisis multivariante de la puntuación del cuestionario KHQ según características y sintomas

\begin{tabular}{lcccc}
\hline & B & Error típico & t-valor & $\mathbf{p}^{(\mathbf{1})}$ \\
\hline Constante & 85,8 & 26,4 & 3,2541 & 0,0012 \\
Edad (años) & $-1,4$ & 0,5 & $-2,7996$ & 0,0053 \\
IMC $\left(\mathrm{Kg} / \mathrm{m}^{2}\right)$ & $-2,8$ & 1,0 & $-2,7798$ & 0,0057 \\
Edad*IMC & 0,1 & 0,0 & 3,3372 & 0,0009 \\
Incontinencia por urgencia ${ }^{(2)}$ & 6,4 & 1,9 & 3,3182 & 0,0010 \\
Incontinencia por el acto sexual $^{(2)}$ & 11,0 & 1,8 & 6,2612 & $<0,0001$ \\
Infecciones urinarias $^{(2)}$ & 5,4 & 1,7 & 3,1522 & 0,0017 \\
Otros problemas urinarios $^{(2)}$ & 7,8 & 3,6 & 2,1338 & 0,0334 \\
\hline
\end{tabular}

(1) P-valor de las variables significativas del modelo $(\mathrm{p}<0,05)$

(2) Variables dummy con valores 0 (ausencia) y 1 (presencia)

tomas "frecuencia" y "urgencia" puede proporcionar una pista de hacia donde dirigir el tratamiento en una mujer con IU de sintomatología múltiple, dado que además son estos los síntomas que más afectación parecen producir en la mujer. En un estudio anterior ${ }^{6}$ realizado en una muestra de 1.732 mujeres que consultaron por IU en una unidad de uroginecología, las frecuencias de presentación de los diferentes síntomas urinarios fueron sensiblemente diferentes: "IU de esfuerzo" (80\%), “urgencia” (67,1\%), "IU de urgencia" (56,4\%), "frecuencia" $(51,2 \%)$ y "nocturia" (43\%). La diferencia probablemente estriba en que en el presente estudio se utilizó como criterio de inclusión el que la paciente tuviera síntoma de urgencia-frecuencia, con o sin IU asociada y además también hay que considerar la diferencia que puede suponer el que en este estudio la información se obtiene a través de un cuestionario cumplimentado por la paciente; mientras que en el estudio referido ${ }^{6}$ la presencia de los sintomas se determinó a través de entrevista con la paciente. Las diferencias entre ambos métodos de obtención de información han sido identificadas en un reciente estudio ${ }^{7}$ en el que se comprobó que los cuestionarios de sintomas auto-cumplimentados tienen mayor correlación con el diagnóstico urodinámico de IU que la que muestran los cuestionarios administrados por entrevista.

Aunque no presenten un patrón claramente lineal, todos los sintomas, excepto el de IU por Esfuerzo, tienden a aumentar en frecuencia con la edad. Esto confirma lo encontrado en el estudio antes referido realizado por nuestro grupo ${ }^{6}$ en el que se encontró mayor frecuencia relativa de síntoma de "IU de esfuerzo" en las mujeres meno- 
res de 65 años mientras que la del resto de síntomas evaluados ("urgencia", "IU de urgencia", "frecuencia" y "nocturia") fue mayor en el grupo de mujeres de 65 o más años.

Es de señalar que una cuarta parte de las mujeres a las que se les entregó el cuestionario no cumplimentaron todos los items. La dimensión con mayor tanto por ciento de mujeres que no respondieron a alguno de sus items fue $\mathrm{Li}$ mitaciones Físicas al igual que ocurrió en el estudio de validación de la versión española ${ }^{4}$, sólo que en el presente estudio el porcentaje fue superior $(8,4 \%$ vs $5,6 \%)$ estando otras tres dimensiones por encima del 6\% (Relaciones Personales, Limitaciones Sociales e Impacto de la Incontinencia Urinaria). En un estudio anterior ${ }^{8}$ se habían identificado dificultades en la respuesta a las preguntas de la dimensión Relaciones Personales del cuestionario KHQ por proponer la categoría "no procede" entre las posibles opciones, dificultades que se observaron también en el presente estudio.

Las puntuaciones medias en las diferentes dimensiones del cuestionario KHQ son similares entre los diferentes grupos según la presencia de síntomas urinarios. Las puntuaciones globales medias del cuestionario fueron también muy parecidas entre los diferentes grupos. En todos los grupos las puntuaciones globales del KHQ fueron superiores (peor calidad de vida) en las pacientes que tenían el síntoma respecto a las que no lo tenían. Aunque al cuestionario $\mathrm{KHQ}$ discrimina perfectamente a las pacientes con sintomas de IU respecto a las que no tienen, no parece existir un perfil de afectación propio de cada síntoma o grupo diagnóstico que justifique un cuestionario tan largo para obtener puntuaciones en diferentes dimensiones. Diferente sería la situación en la que cada grupo sintomático evidenciara alteraciones diferentes en las dimensiones del cuestionario (por ejemplo: Urgencia, mayor impacto en actividades de la vida cotidiana que los otros grupos de síntomas, que permitiría disponer de una medida secundaria para evaluación de resultados de posibles intervenciones sobre el sintoma).

La falta de poder discriminatorio entre los diferentes sintomas, a pesar de la longitud del cuestionario, probablemente hace deseable la utilización, en la práctica clínica, de cuestionarios más breves para estudiar el impacto que la IU produce en la calidad de vida, reservando el cuestionario KHQ para investigación.

Los síntomas que más contribuyen a la afectación de la calidad de vida, de acuerdo al análisis multivariante presentado en la Tabla 4, son la "incontinencia en el acto sexual", "otros problemas urinarios" (epígrafe bajo el que se agrupan las diferentes respuestas a la correspondiente pregunta con respuesta abierta del cuestionario), la "incontinencia por urgencia-IUU" y las "infecciones frecuentes en vías urinarias". Lo evidenciado a través de este modelo es que la IUU parece tener mayor influencia en la calidad de vida que la IU de Esfuerzo y otros sintomas clásicos de IU, hecho ya descrito previamente ${ }^{9}$. Llama la atención en este punto la asociación independiente de la "incontinencia en el acto sexual" con la puntuación total del cuestionario KHQ. En realidad lo que nos muestra el modelo es que las mujeres con "IU en el acto sexual" vs las que no la tienen presentan puntuaciones totales medias del $\mathrm{KHQ}$ claramente superiores $(46,8$ vs 33,1$)$. Teniendo en cuenta que las mujeres con este problema tendrán puntuaciones muy altas en la dimensión "Relaciones Personales-RP" del KHQ, (cuyas preguntas ahondan en este aspecto de la calidad de vida) no es de extrañar que tengan también una elevada puntuación total del KHQ, lo que explicaría, al menos en parte, la presencia de esta fuerte asociación. Es necesario profundizar en la investigación de la afectación que la IU y el síndrome de VH causan en la esfera de la vida de pareja de la mujer. La afectación de la función sexual por la IU ya ha sido descrito previamente ${ }^{10}$ y aunque según algunos autores, esta afectación no se produciria en el síndrome de vejiga hiperactiva sin $\mathrm{IU}^{11}$, consideramos que la presencia de síntomas como la urgencia y frecuencia puede alterar el ambiente de pareja propicio al contacto sexual.

\section{REFERENCIAS}

1. Donovan J, Bosch R. Symptoms and quality of life assessment. In Abrams P, Cardozo L, Khoury S, Wein A. Incontinence. 3rd International Consultation on Urinary Incontinence. Edition 2005. Plymbridge, Mass: Health Publication Ltd. United Kingdom, ISBN 0- 9546956-2-3. 
2. Jolleys JV, Donovan Jl, Nanchahal K, Peters TJ, Abrams P. Urinary symptoms in the community: How bothersome are they? Br J Urol. 1994;74(5):551-555.

3. Espuña M. Incontinencia de orina en la mujer. Med Clín (Barc). 2003;120(12):464-472.

4. Badía X, Castro D, Conejero J. Validez del cuestionario King's Health Questionnaire para la evaluación de la calidad de vida de los pacientes con incontinencia urinaria. Med Clin (Barc). 2000; 114(5):647-652.

5. Kelleher CJ, Cardozo LD, Khullar V, Salvatore S. A new questionnaire to assess the quality of life of urinary incontinent women. British Journal of Obstetrics and Gynaecology. 1997;104(12):1374-1379.

6. Espuña M, Puig M, Pérez A, Rebollo P. Incontinencia de orina de esfuerzo: primera causa de incontinencia en las mujeres remitidas a una unidad de uroginecología. Arch Esp Urol. 2004;57(6):633-640.

7. Khan MS, Chalina C, Leskova L, Khullar V. The relationship between urinary symptom questionnaires and urodynamic diagnoses: an analysis of two methods of questionnaire administration. BJOG 2004;111(5):468-474.
8. Reese PR, Pleil AM, Okano GJ, Kelleher CJ. Multinational study of reliability and validity of the King's Health Questionnaire in patients with overactive bladder. Qual Life Res. 2003;12(4):427-442.

9. Palmtag H. The patient's perspective: redefining end points. Urology 2004;64(suppl.1):17-20.

10. Yip SK, Chan A, Pang S, Leung P, Tang C, Shek D, Chung $\mathrm{T}$ The impact of urodynamic stress incontinence and detrusor overactivity on marital relationship and sexual function. Am J Obstet Gynecol. 2003;188(5):1244-1248.

11. Coyne K, Revicki D, Hunt T, Corey R, Stewart W, Bentkover J, Kurth H, Abrams P. Psychometric validation of an overactive bladder symptom and health-related quality of life questionnaire: the OAB-q. Qual Life Res. 2002;11(6):563-574.

Dra. M. Espuña Pons

ICGON. Hospital Clínic

Villarroel 170 - 08036 Barcelona

12922mep@comb.es

(Trabajo recibido el 11 de febrero 2006) 